\title{
Segmental infarction of the omentum: diagnosis by ultrasound
}

\author{
V. Naraynsingh, R. Barrow, G.C. Raju and L.U. Manmohansingh \\ Departments of Surgery and Radiology, Port-Of-Spain General Hospital, Trinidad, West Indies
}

\begin{abstract}
Summary: Pre-operative diagnosis of segmental infarction of the omentum is difficult because of its rarity and non-specific clinical features. We present a case of omental infarction and suggest that ultrasound scan may be helpful in the diagnosis of this entity.
\end{abstract}

\section{Introduction}

Segmental infarction of the omentum is very rare. Since its first description by Bush in 1896, fewer than 100 cases have been reported (Epstein \& Lempke, 1968). Because of its rarity and non-specific clinical features the diagnosis is not usually made pre-operatively. We describe a case in which the diagnosis was suggested by ultrasound and confirmed at laparotomy.

\section{Case report}

A 33 year old medium built, East Indian male, presented with a 10 day history of left upper abdominal pain. He was under medical supervision for that time but $2 \mathrm{~d}$ before admission he was noticed to have a mass in the left upper quadrant of the abdomen and mild pyrexia. This constant pain did not radiate but became progressively worse until, on admission, it was aggravated by coughing and movement. He had no nausea, vomiting, anorexia, diarrhoea, constipation or urinary symptoms.

He was a well looking anicteric man with a mild pyrexia $\left(37.2^{\circ} \mathrm{C}\right)$. The only significant finding was a firm tender immobile mass in the left upper quadrant of the abdomen, $8 \times 5 \mathrm{~cm}$, which extended below and medial to the left costal margin. The haemoglobin and white cell count were within normal limits. Although there was no history of trauma, the possibility of a tender splenic mass was entertained. Ultrasound scan revealed that the mass was situated inferior and lateral to the greater curve of the stomach when the latter was water-filled. The left kidney, spleen and pancreas were normal and not related to the mass which was complex and non-homogeneous with a mixture of solid material and ill-defined hypoechoic zones (Figure 1).

Careful monitoring during real time sector scanning failed to show any internal movement that might

Correspondence: V. Naraynsingh, F.R.C.S.

Accepted: 13 December 1984 suggest the lumen of the gut. Anatomically the $7 \times 4 \mathrm{~cm}$ mass was identified as lying between the greater curvature of the stomach and transverse colon and probably represented necrotic omentum or an organizing haematoma.

At laparotomy, an omental mass, lying between the greater curvature of the stomach and transverse colon, was adherent to the anterior abdominal wall. This haemorrhagic mass with necrotic areas was easily separated from the abdominal wall, transverse colon and stomach, and excised. Areas of haemorrhage and necrosis were visible both on the surface and cut

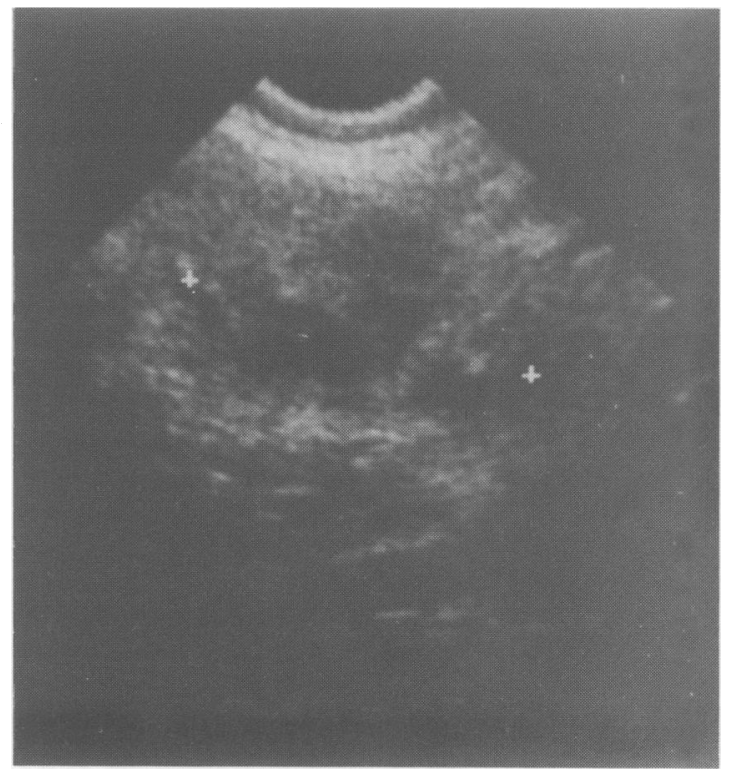

Figure 1 Ultrasound scan of mass $(+\ldots+)$ showing solid areas and hypoechoic zones.

(C) The Fellowship of Postgraduate Medicine, 1985 
section of this $7 \times 4 \mathrm{~cm}$ omental mass (Figure 2). Histology confirmed infarction and haemorrhage in an otherwise normal omentum.

\section{Discussion}

Segmental infarction of the omentum can occur at any age but usually presents in young and middle-aged adults (Epstein \& Lempke, 1968; Kakar \& Taneja, 1980; Halligan \& Rabiah, 1959). Pain and vomiting, the commonest symptoms, are so non-specific that the diagnosis is not usually suspected. A palpable mass is present in about $50 \%$ of children but is less common in adults (Martorell, 1968). The pathogenesis of this condition is not clear but it is known to occur with or without torsion of the omentum (Vertuno et al., 1980). In our patient there was no torsion as the affected omentum lay in its normal anatomical position between the stomach and transverse colon. Though rare, it is important to recognize this condition since it can mimic other common and grave causes of the acute abdomen (Kakar \& Taneja, 1980). Our case demonstrates for the first time that ultrasound may be used safely and effectively to identify segmented infarction of the omentum. It must be remembered, however, that abscesses in the omentum, arising from adjacent colonic or gastric lesions, may resemble omental infarction on ultrasound scan and surgical exploration remains the safest approach to the patient with this condition.

\section{References}

BUSH, P. (1896). A case of haemorrhage into the greater omentum. Lancet, i, 286.

EPSTEIN, L.I. \& LEMPKE, R.E. (1968). Primary idiopathic segmental infarction of the greater omentum. A collective review and report of a case. Annals of Surgery, 167, 437.

HALLIGAN, E.J. \& RABIAH, F.A. (1959). Primary idiopathic segmental infarction of the greater omentum. Archives of Surgery, 79, 738.

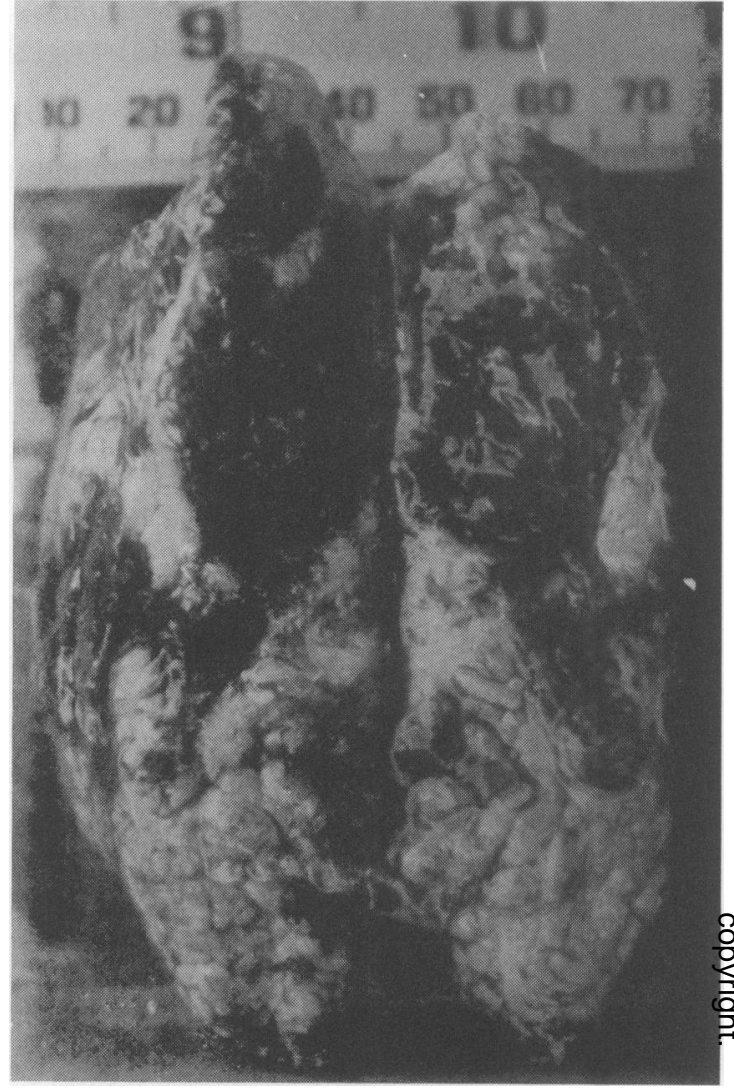

Figure 2 Omental mass showing areas of haemorrhage and necrosis on the cut surface.

KAKAR, A. \& TANEJA, S.B. (1980). Infarction of the greater omentum. Diseases of the Colon and Rectum, 23, 430.

MARTORELL, R.A. (1968). Idiopathic torsion and infarction of the omentum. American Surgeon, 34, 252.

VERTUNO, L.L., DAN, J.R. \& WOOD, W. (1980). Segmental infarction of the omentum. American Journal of Gastroenterology, 74, 443. 